EPJ Web of Conferences 71, 00108 (2014)

DOI: $10.1051 /$ epjconf/20147100108

(C) Owned by the authors, published by EDP Sciences, 2014

\title{
Recent BABAR results on dark matter and light Higgs searches and on CP and T violation
}

\author{
Ida Peruzzi, representing the BABAR Collaboration \\ ${ }^{1}$ Laboratori Nazionali di Frascati dell'INFN, Via E. Fermi 40, Frascati, Italy \\ and also \\ Dipartimento di Fisica dell'Università di Perugia, Via A. Pascoli, Perugia, Italy
}

\begin{abstract}
Recent BABAR results on several topics are presented. We report the observation of T-violation as well as recent results on CP-violation in $\mathrm{B}$ decays in a dataset collected at the $\mathrm{Y}(4 \mathrm{~S})$ resonance. We also present searches for low mass CP-odd Higgs bosons, predicted in non-minimal SUSY models, as well as searches for dark-sector gauge bosons motivated by astrophysical observations, using radiative decays of the $\mathrm{Y}(1 \mathrm{~S}, 2 \mathrm{~S}, 3 \mathrm{~S})$ resonances and other $e^{+} e^{-}$processes. These searches enable stringent limits to be set on the parameters of such particles.
\end{abstract}

\section{Introduction}

BABAR [1] is a detector optimized for CP Violation (CPV) studies of the B system produced in $e^{+} e^{-}$by the boosted $\Upsilon(4 S)$ resonance. The data taking at the SLAC PEP-II asymmetric collider started in 2000 and ended in April 2008; a great number of events on the Y resonances was recorded, and many analyses are still going on, producing refinements of previous work or completely new results. The spectrum of BABAR Physics is very wide, having to pick a few topics for this Conference, I chose the measurement of $\mathrm{T}$ violation in $B^{0}$ decays, which was made possible following an original idea, and some searches for hypothetical particles suggested in many model beyond the Standard Model, as light Higgs or dark matter particles.

\section{$2 \mathrm{CP}$ and Time Reversal Violation}

\subsection{Data Sample}

The CP Violazion (CPV) and the Time Reversal Violation (TRV) analysis make use of the full dataset recorded by the BABAR detector at the PEP-II machine at SLAC . This sample consists of $426 \mathrm{fb}^{-1}$ integrated luminosity at the $\Upsilon(4 S)$ resonance, corresponding to $468 \times 10^{6} B \bar{B}$ pairs, and $45 \mathrm{fb}^{-1}$ recorded at a center of mass $(\mathrm{CM})$ energy $40 \mathrm{MeV}$ below the $\Upsilon(4 S)$ - the "offpeak sample" used for background studies. The TRV analysis uses the same $B$ meson samples as our most recent timedependent $C P$ asymmetry measurement in $b \rightarrow c \bar{c} s$ decays [2] - with the exception of the $\eta_{c} K_{s}^{0}$ and $J / \psi K^{* 0}$ final states - as well as the same reconstruction algorithms, selection criteria, and calibration techniques.

This is an Open Access article distributed under the terms of the Creative Commons Attribution License 2.0, which permits unrestricted use, distribution, and reproduction in any medium, provided the original work is properly cited. 


\subsection{Time Reversal Violation: the experimental problem}

I will start by recalling what testing the basic symmetries in a process means: application of $\mathrm{CP}$ changes all particles to their antiparticles: CP violation implies $i \rightarrow f \neq \bar{i} \rightarrow \bar{f}$; the effect of $\mathrm{T}$ is the exchange of final and initial states :TRV implies $i \rightarrow f \neq f \rightarrow i$ (with time inversion ). The Standard Model is a local gauge theory and this requires, on very solid grounds, CPT invariance. Experimentally it has been proven that CP is violated in neutral Kaon and B systems, so we have indirect proof of $\mathrm{T}$ violation. The question is: can we prove $\mathrm{T}$ violation without assuming CPT invariance? CPLEAR [3] claims to have proven T violation in $K^{0}$ mixing, but this is controversial because in the mixing the effect of $\mathrm{CP}$ and $\mathrm{T}$ is the same, a part the time inversion, which is tricky. In the last decade BABAR (and Belle) have measured many effects of CP violation, in the direct decay and in the interference between mixing and decay of $B^{0} \mathrm{~s}$ : the problem is that to prove T violation we should be able to invert one of these decays, and this is not experimentally feasible!

\subsection{The Time reversal measurement: the solution}

Before offering the solution to the problem, I will recall how $\mathrm{CP}$ is measured by BABAR and Belle, based on the so called quantum entanglement in the $\Upsilon(4 S)$ decay to a pair of neutral B mesons. The $\Upsilon(4 S)$ has the same quantum numbers of the photon, and the two $B^{0}$ must oscillate in a coherent way until one of them decays. At that instant, that we call $t_{\text {tag }}$, the other B must the in the opposite flavor state. As a consequence, the decay of one B to a flavor definite final state fixes the flavor of the other B at time $t_{\text {tag }}$; the second B will then decay after a time $t$; if completely reconstructed into a CP eigenstate, we will call it $B_{\text {rec }}$, decaying at time $t_{r e c}$ with $t=t_{r e c}-t_{\text {tag }}$. We measure the distance between the 2 decay vertices, approximated by the difference of the $\mathrm{z}$ coordinates, and from that we deduce the time difference $t$. The clock starts with the decay of the flavor $\operatorname{tag} B_{t a g}$, so the time $t$ is positive if $B_{r e c}$ decays after $B_{t a g}$, negative when $B_{r e c}$ is the first to decay. The most precise measurements have been made in the golden channel $B \rightarrow J / \psi K_{S}^{0}$, the CP asymmetry has also been measured in $B \rightarrow J / \psi K_{L}^{0}$.

The method to independently measure TRV was first suggested in [4] and then applied specifically [5] to the BABAR case; it is basically the observation that the EPR applies to any pair of orthogonal states, not just flavor pairs. If we can consider a $B^{0} \mathrm{CP}$ odd and $B^{0} \mathrm{CP}$ even pair $|i n\rangle$ of the two $B$ mesons produced in the $\Upsilon(4 S)$ decay, the state can be written as:

$$
\begin{aligned}
& \mid \text { in }\rangle=\frac{1}{\sqrt{2}}\left[B^{0}\left(t_{1}\right) \bar{B}^{0}\left(t_{2}\right)-\bar{B}^{0}\left(t_{1}\right) B^{0}\left(t_{2}\right)\right], \quad \text { or } \\
& \mid \text { in }\rangle=\frac{1}{\sqrt{2}}\left[B_{+}\left(t_{1}\right) B_{-}\left(t_{2}\right)-B_{-}\left(t_{1}\right) B_{+}\left(t_{2}\right)\right] .
\end{aligned}
$$

Here $B^{0}$ and $\bar{B}^{0}$ indicate the usual flavor states, $B_{+}$and $B_{-}$are orthogonal $B$ states with definite $C P$, and $t_{1}$ and $t_{2}$ are the decay times of these states. The first line of eqn. 1 as been used at the $B$ factories for CPV studies; the second line introduces " $C P$ tagging": observation at time $t_{1}$ of a final state with positive $C P$ implies that at that time the other $B$ was in a $C P=-1$ state. B flavor tagging has been implemented by BABAR and Belle using sophisticated analysis techniques and several categories of decay modes; in the following we will use the simple notations $B^{0} \Leftrightarrow \ell^{+}, \bar{B}^{0} \Leftrightarrow \ell^{-}$to indicate the $B$ flavor from the sign of the decay lepton of the other $B$.

Using a combination of flavor and CP tagging it's possible to build T reversed events. In $B$ meson decays to $c \bar{c} s$ such as $B \rightarrow J / \psi K^{0}$, the final state $J / \psi K_{S}^{0}$ is $C P$ odd, while $J / \psi K_{L}^{0}$ has positive $C P$ 
eigenvalue, we therefore identify the $C P$ states according to the final state of the other $B$ meson: $B_{+} \Leftrightarrow K_{S}^{0}, B_{-} \Leftrightarrow K_{L}^{0}$.

Preparing a T reverse event is now possible! If, for example, we have one event where the first decay is identified by flavor tag as a $B^{0}$ and the later decay is a $J / \psi K_{L}^{0}$, we have the process: $\bar{B}^{0} \rightarrow B_{+}$, with positive decay time. The T reverse event would be a decay into $J / \psi K_{S}^{0}$, which tags the other B as a $B_{+}$at time $t_{1}$; if this later decays into a negative lepton, showing its flavor as a $\bar{B}^{0}$ we have the process: $B_{+} \rightarrow \bar{B}^{0}$, with negative decay time.

In the following, in the event sample selected in the $\sin (2 \beta)$ measurements, we will denote a process $\left(\ell^{-}, B^{0} \rightarrow B_{+}\right)$as $\left(\ell^{-}, K_{L}^{0}\right)$ if one of the $B$ mesons decays to a final state with definite flavor $\left(e . g . \ell^{-}\right)$and the other to a state with definite $C P\left(e . g . K_{L}^{0}\right)$. By suitably exchanging $B_{+}, B_{-}, K_{s}^{0}, K_{L}^{0}$ in the time-ordered decay sequence, it is possible to construct the $T$-transformed decay processes (exchange of the initial and final states), detailed in Table 1.

\begin{tabular}{cc|cc}
\hline \hline $\begin{array}{c}\text { Reference } \\
\text { transition }\end{array}$ & $\begin{array}{c}\text { Time ordered } \\
\text { event }\end{array}$ & $\begin{array}{c}T \text {-transformed } \\
\text { transition }\end{array}$ & $\begin{array}{c}\text { Time ordered } \\
\text { event }\end{array}$ \\
\hline$B^{0} \rightarrow B_{+}$ & $\left(\ell^{-}, K_{L}^{0}\right)$ & $B_{+} \rightarrow B^{0}$ & $\left(K_{S}^{0}, \ell^{+}\right)$ \\
$\bar{B}^{0} \rightarrow B_{+}$ & $\left(\ell^{+}, K_{L}^{0}\right)$ & $B_{+} \rightarrow \bar{B}^{0}$ & $\left(K_{S}^{0}, \ell^{-}\right)$ \\
$B^{0} \rightarrow B_{-}$ & $\left(\ell^{-}, K_{S}^{0}\right)$ & $B_{-} \rightarrow B^{0}$ & $\left(K_{L}^{0}, \ell^{+}\right)$ \\
$\bar{B}^{0} \rightarrow B_{-}$ & $\left(\ell^{+}, K_{S}^{0}\right)$ & $B_{-} \rightarrow \bar{B}^{0}$ & $\left(K_{L}^{0}, \ell^{-}\right)$ \\
\hline \hline
\end{tabular}

Table 1. Reference and $T$-transformed $B$-meson transitions. As discussed in the text, $\left(\ell^{-}, K_{L}^{0}\right)$ for the transition $B^{0} \rightarrow B_{+}$is a short-hand notation for $\left(\ell^{-} X, J / \psi K_{L}^{0}\right)$, and similarly for the other decay modes. For each transition, the first of the final states in parenthesis is the one from the earlier $B$ decay.

Any difference between the reference and the $T$-reversed rates would be an evidence of $T$ symmetry violation.

The eight transitions of Table 1 have a time-dependent decay rate given by:

$$
g_{\alpha, \beta}^{ \pm}(\Delta \tau) \propto e^{-\Gamma_{d} \Delta \tau}\left\{1+S_{\alpha, \beta}^{ \pm} \sin \left(\Delta m_{d} \Delta \tau\right)+C_{\alpha, \beta}^{ \pm} \cos \left(\Delta m_{d} \Delta \tau\right)\right\}
$$

where indices $\alpha=\ell^{+}, \ell^{-}$and $\beta=K_{S}^{0}, K_{L}^{0}$ indicate the flavor- and $C P$ - tagged final states, respectively, and the symbol + or - indicates whether the decay to the flavor final state $\alpha$ occurs before or after the decay to the $C P$ final state $\beta$. In eqn. $2, C_{\alpha, \beta}^{ \pm}, S_{\alpha, \beta}^{ \pm}$are 16 model independent coefficients, related to $C P$ violation, that can be measured. In the Standard Model all $C_{\alpha, \beta}^{ \pm}$are expected to be zero, and $S_{\alpha, \beta}^{ \pm}=\sin (2 \beta) . T$ violation would manifest itself through differences between the $C_{\alpha, \beta}^{ \pm}$or $S_{\alpha, \beta}^{ \pm}$values for $T$-conjugated processes.

\subsection{The BABAR Measurement}

The afore mentioned method has been applied by BABAR [6] to measure TRV, making use of same events selected in the previous $\sin 2 \beta$ measurements in $B$ meson decays to $c \bar{c}$ [2].

The composition of the final sample is obtained from fits to the distributions of $m_{\mathrm{ES}}$ for $c \bar{c} K_{S}^{0}$ and of $\Delta E$ for $J / \psi K_{L}^{0}$, using parametric forms and distributions extracted from MC simulation and $J / \psi$ dilepton mass sidebands in data to describe the signal and background components (see Fig. 1). The final sample contains $7796 c \bar{c} K_{s}^{0}$ events, and $5813 \mathrm{~J} / \psi K_{L}^{0}$ events. In the signal region, defined respectively as

$$
\begin{array}{ll}
m_{\mathrm{ES}} \in[5.27,5.29] \mathrm{GeV} / c^{2} & \left(c \bar{c} K_{S}^{0}\right) \\
|\Delta E|<10 \mathrm{MeV} & \left(J / \psi K_{L}^{0}\right)
\end{array}
$$



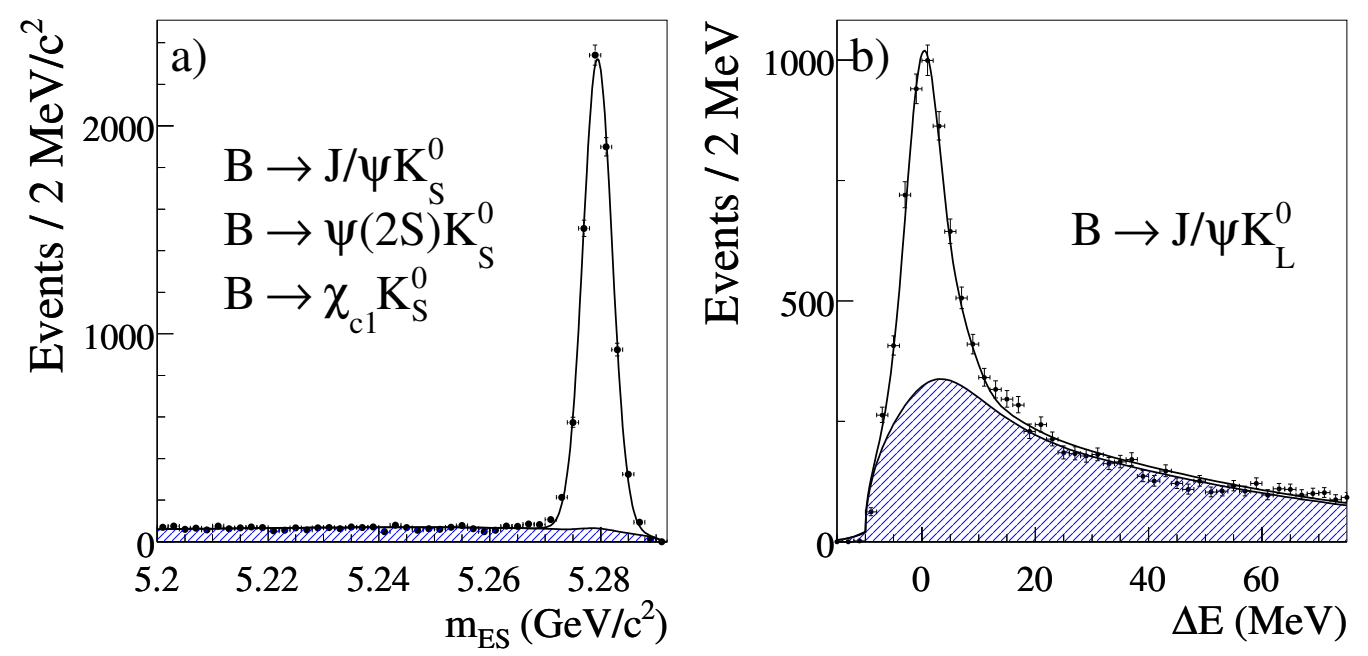

Figure 1. Distributions of $m_{\mathrm{ES}}$ (a) and $\Delta E$ (b) for the $c \bar{c} K_{S}^{0}$ and $J / \psi K_{L}^{0}$ samples respectively. The shaded areas represent the estimated background contributions.

the purity ranges from $87 \%$ and $96 \%$ for the various categories of $c \bar{c} K_{S}^{0}$ events, and is $56 \%$ for $J / \psi K_{L}^{0}$ events.

Events are assigned signal and background probabilities based on the $m_{\mathrm{ES}}$ or $\Delta E$ distributions. An independent, large statistics sample mainly composed by $B^{0} \rightarrow D^{(*)-} X$ decays is used to determine on data the vertex resolution function and the mistag probability. The $C_{\alpha, \beta}^{ \pm}, S_{\alpha, \beta}^{ \pm}$coefficients are determined through a simultaneous unbinned maximum likelihood fit to the $\Delta t$ distributions for $c \bar{c} K_{s}^{0}$ and $J / \psi K_{L}^{0}$ events, split by flavor category.

The signal probability density function $(\mathrm{PDF})$ is given by [5]:

$$
\begin{aligned}
\mathcal{H}_{\alpha, \beta}(\Delta t) & \propto g_{\alpha, \beta}^{+}\left(\Delta t_{\text {true }}\right) H\left(\Delta t_{\text {true }}\right) \otimes \mathcal{R}\left(\delta t ; \sigma_{\Delta t}\right) \\
& +g_{\alpha, \beta}^{-}\left(-\Delta t_{\text {true }}\right) H\left(-\Delta t_{\text {true }}\right) \otimes \mathcal{R}\left(\delta t ; \sigma_{\Delta t}\right) .
\end{aligned}
$$

Here $\Delta t$ is the signed proper time difference between the two $B$ decays, determined from the $B$ meson vertex separation projected along the collision axis; $\Delta t_{\text {true }}$ is the same variable in the limit of perfect reconstruction; $\delta t \equiv \Delta t-\Delta t_{\text {true }}$, and $H$ the Heaviside step function, needed to separate events with $\Delta t>$ 0 and $\Delta t<0$. Vertex resolution effects are accounted for by convolving with the resolution function $\mathcal{R}\left(\delta t ; \sigma_{\Delta t}\right)$. A non-zero probability of assigning the wrong flavor tag, $\omega$, reduces the sensitivity to the $\left(C_{\alpha, \beta}^{ \pm}, S_{\alpha, \beta}^{ \pm}\right)$parameters by a factor $(1-2 \omega)$ (not shown in Eq. 2). A total of 27 parameters are varied in the likelihood fit: eight pairs of $\left(C_{\alpha, \beta}^{ \pm}, S_{\alpha, \beta}^{ \pm}\right)$coefficients for the signal, and 11 parameters describing possible $C P$ and $T$ violation in the background.

\subsection{Results}

From the 16 signal coefficients $\left(C_{\alpha, \beta}^{ \pm}, S_{\alpha, \beta}^{ \pm}\right)$we construct six independent pairs of asymmetry parameters $\left(\Delta C_{T}^{ \pm}, \Delta S_{T}^{ \pm}\right),\left(\Delta C_{C P}^{ \pm}, \Delta S_{C P}^{ \pm}\right)$and $\left(\Delta C_{C P T}^{ \pm}, \Delta S_{C P T}^{ \pm}\right)$, explicitly defined in the second column of Table 2. $T$-symmetry breaking would directly manifests itself through any nonzero value of $\Delta S_{T}^{ \pm}$or $\Delta C_{T}^{ \pm}$, and analogously for $C P$ - or $C P T$-symmetry breaking. 
The measured values [6] for the asymmetry parameters are reported in Table 2; the first error is the fit statistical uncertainty.

\begin{tabular}{|c|c|c|}
\hline Parameter & Definition & Result \\
\hline$S_{\rho^{+} K^{0}}^{+}$ & & $0.55 \pm 0.09 \pm 0.06$ \\
\hline$S^{\underline{\ell}^{+}, K_{S}^{0}}$ & & $-0.66 \pm 0.06 \pm 0.04$ \\
\hline$C_{\rho^{+}}^{\ell_{+}^{+}, K_{S}^{0}}$ & & $0.01 \pm 0.07 \pm 0.05$ \\
\hline$C_{\ell^{+}, K_{S}^{0}}^{-, n_{S}}$ & & $-0.05 \pm 0.06 \pm 0.03$ \\
\hline$\Delta S_{T}^{+}$ & $S_{\ell^{-}, K^{0}}^{-}-S_{\ell^{+}, K_{s}^{0}}^{+}$ & $-1.37 \pm 0.14 \pm 0.06$ \\
\hline$\Delta S_{T}^{-}$ & 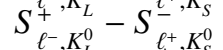 & $1.17 \pm 0.18 \pm 0.11$ \\
\hline$\Delta C_{T}^{+}$ & $C_{\ell^{-}, K_{L}^{0}}^{-n_{L}}-C_{\ell^{+}, K_{S}^{0}}^{+, n_{S}}$ & $0.10 \pm 0.14 \pm 0.08$ \\
\hline$\Delta C_{T}^{-}$ & $C_{\ell^{-}, K_{L}^{0}}^{+, h_{L}}-C_{\ell^{+}, K_{S}^{0}}^{-,_{S}}$ & $0.04 \pm 0.14 \pm 0.08$ \\
\hline$\Delta S_{C P}^{+}$ & $S_{\ell^{-}, K_{S}^{0}}^{+}-S_{\ell^{+}, K_{S}^{0}}^{+}$ & $-1.30 \pm 0.11 \pm 0.07$ \\
\hline$\Delta S_{C P}^{-}$ & 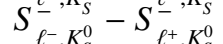 & $1.33 \pm 0.12 \pm 0.06$ \\
\hline$\Delta C_{C P}^{+}$ & $C_{\ell^{-}, K_{S}^{+}}^{+}-C_{\ell^{+}, K_{S}^{0}}^{+, K_{S}}$ & $0.07 \pm 0.09 \pm 0.03$ \\
\hline$\Delta C_{C P}^{-}$ & $C_{\ell^{-}, K_{S}^{0}}^{-, n_{S}}-C_{\ell^{+}, K_{S}^{0}}^{-, n_{S}^{0}}$ & $0.08 \pm 0.10 \pm 0.04$ \\
\hline$\Delta S_{C P T}^{+}$ & $S_{\rho^{+} K^{0}}^{-}-S_{\rho^{+}}^{+}$ & $0.16 \pm 0.21 \pm 0.09$ \\
\hline$\Delta S_{C P T}^{-}$ & $S_{\rho^{+} K^{+}}^{+, K_{L}}-S_{\rho^{+} K^{0}}^{-, K_{S}}$ & $-0.03 \pm 0.13 \pm 0.06$ \\
\hline$\Delta C_{C P T}^{+}$ & $C_{\ell^{+}, K^{0}}^{-, K_{L}}-C_{\ell^{+}, K_{S}^{0}}^{+, K_{S}^{0}}$ & $0.14 \pm 0.15 \pm 0.07$ \\
\hline$\Delta C_{C P T}^{-}$ & $C_{\ell^{+}, K_{L}^{0}}^{+, n_{L}}-C_{\ell^{+}, K_{S}^{0}}^{-,_{S}}$ & $0.03 \pm 0.12 \pm 0.08$ \\
\hline
\end{tabular}

Table 2. Measured values of the $T$-, $C P$-, and $C P T$-asymmetry parameters, defined as the differences in $S_{\alpha, \beta}^{ \pm}$ and $C_{\alpha, \beta}^{ \pm}$between symmetry-transformed transitions. The values of reference coefficients are also given at the top. The first uncertainty is statistical and the second systematic. The indices $\ell^{-}, \ell^{+}, K_{S}^{0}$, and $K_{L}^{0}$ stand for reconstructed final states that identify the $B$ meson as $\bar{B}^{0}, B^{0}, B_{-}$, and $B_{+}$, respectively.

Systematic uncertainties are evaluated in a similar way as in the BABAR $C P$ analysis of $B \rightarrow$ $c \bar{c} K^{(*) 0}$ decays [2]. The dominant uncertainties arise from the imperfect description of the $\Delta t$ resolution and of the $J / \psi K_{L}^{0}$ background. A central assumption in the analysis is that $c \bar{c} K_{S}^{0}$ and $J / \psi K_{L}^{0}$ are orthogonal states, which is not valid in principle if the $C P$ symmetry is not conserved in the $K^{0} \bar{K}^{0}$ system or in $B$ decays. However, the former is a $O\left(10^{-3}\right)$ effect, and the latter is theoretically expected to be very small in $b \rightarrow c \bar{c} s$ decays, and experimentally confirmed by the measured values of the $C_{\alpha, \beta}^{ \pm}$ coefficients, consistent with zero. In either case the effect is well below the statistical uncertainty.

A number of consistency checks were performed to validate the result: repeating the analysis as in the standard $C P$ analysis we obtain the same values and uncertainties as those in [2] for the single pair of $(C, S)$ parameters from the fit; replacing the $c \bar{c} K_{S}^{0}$ final state with $c \bar{c} K^{ \pm}$and $J / \psi K_{L}^{0}$ with $J / \psi K^{* \pm}$ yields as expected a null result.

Violation of the $T$ reversal invariance can be visually demonstrated by constructing the timedependent asymmetry

$$
A_{T}\left(\Delta t^{+}\right) \simeq \frac{\Delta S_{T}^{+}}{2} \sin \left(\Delta m_{d} \Delta t\right)+\frac{\Delta C_{T}^{+}}{2} \cos \left(\Delta m_{d} \Delta t\right)
$$

and similarly for the other three $T$-violating asymmetries. Figure 2 shows the four asymmetries overlaid with the projection of the best fit results to the $\Delta t$ distributions in the nominal fit (solid red line) 
and when the eight $T$-invariance constrains:

$$
\Delta S_{T}^{ \pm}=\Delta C_{T}^{ \pm}=0 ; \quad \Delta S_{C P}^{ \pm}=\Delta S_{C P T}^{ \pm} ; \quad \Delta C_{C P}^{ \pm}=\Delta C_{C P T}^{ \pm}
$$

are imposed (dashed blue line). The latter is clearly non compatible with the experimental points.
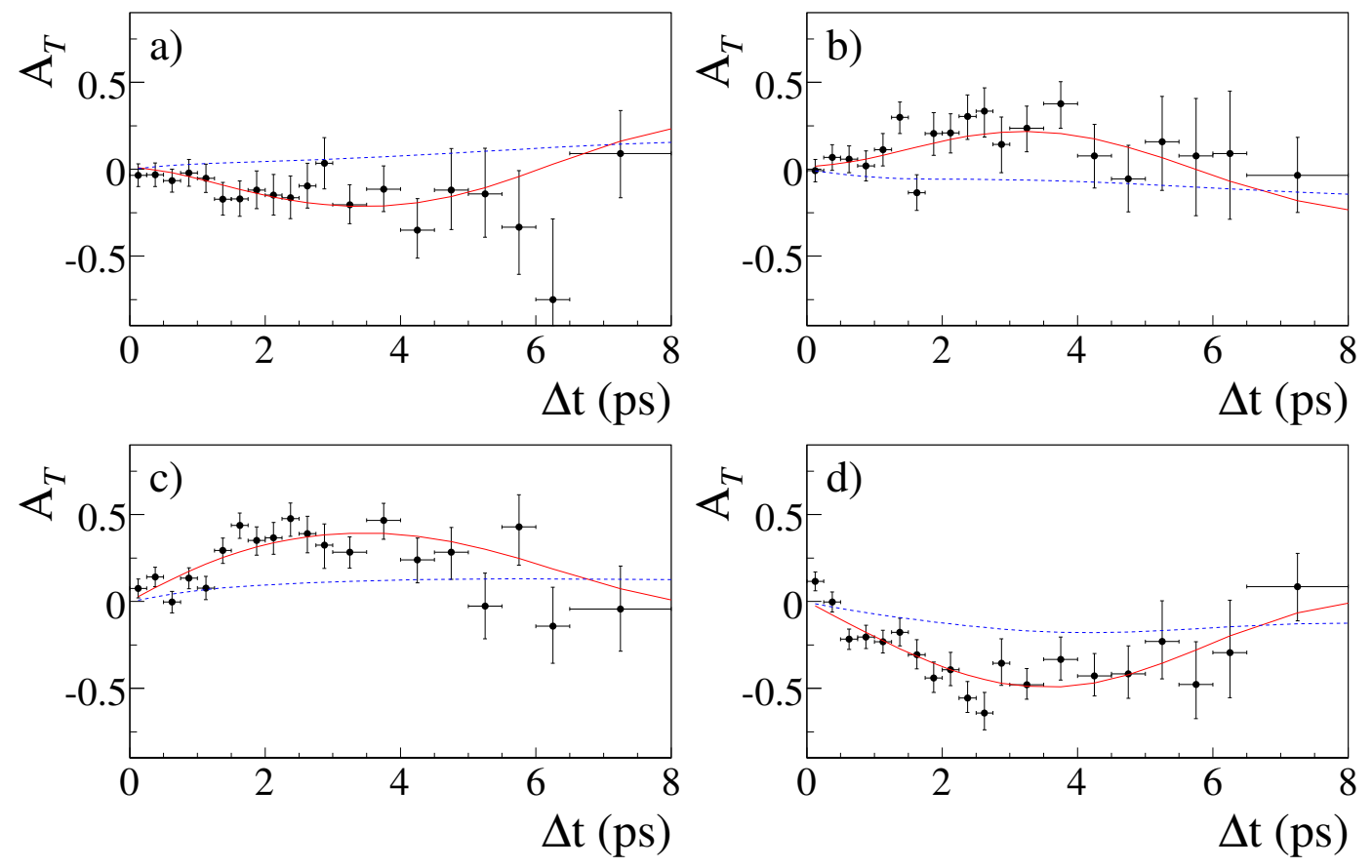

Figure 2. The four independent $T$-asymmetries associated to the reference transitions a) $\bar{B}^{0} \rightarrow B_{-}$, b) $B_{+} \rightarrow B^{0}$, c) $\bar{B}^{0} \rightarrow B_{+}$and d) $B_{-} \rightarrow B^{0}$. The points with error bars represent the data, the red solid and dashed blue curves represent the projections of the best fit results with and without $T$ violation respectively. The dashed blue curves are not perfectly horizontal due to $\Delta t$ resolution effects.

A graphical representation of the $T$-violation result is shown by the two-dimensional confidence level contours in the $\left(\Delta C_{T}^{+}, \Delta S_{T}^{+}\right)\left(\Delta C_{T}^{-}, \Delta S_{T}^{-}\right)$planes in Fig. 3, in which the fit parameters are varied by their statistical and systematic uncertainties.

We evaluate the significance of the $T$-violation signal based on the change in log-likelihood $\left(-2 \Delta \ln \mathcal{L}=-2\left(\ln \mathcal{L}_{n o T R V}-\ln \mathcal{L}\right)\right)$ between the nominal fit and the one where, imposing the constrains of Eqn. 6, TRV is not allowed. In this procedure the statistical and systematic uncertainties are combined, and we find $-2 \Delta \ln \mathcal{L}=226$ with 8 degrees of freedom. Assuming Gaussian errors, this corresponds to a significance equivalent to 14 standard deviations $(\sigma)$, and thus constitutes direct observation ot $T$ violation. We carry out the same procedure for the $\left(\Delta C_{C P}^{ \pm}, \Delta S_{C P}^{ \pm}\right)$variables, finding a significance of $17 \sigma$ thus confirming $C P$ violation, and for the $\left(\Delta C_{C P T}^{ \pm}, \Delta S_{C P T}^{ \pm}\right)$variables, with a significance of $0.3 \sigma$, consistent with $C P T$ invariance.

In summary, the results $\Delta S_{T}^{+}=-1.37 \pm 0.14 \pm 0.06$ and $\Delta S_{T}^{-}=1.17 \pm 0.18 \pm 0.11$ obtained by BABARr comparing the probabilities of $\bar{B}^{0} \rightarrow B_{-}, B_{+} \rightarrow B^{0}, \bar{B}^{0} \rightarrow B_{+}$, and $B_{-} \rightarrow B^{0}$ transitions, to their $T$ conjugate constitute the first observation of $T$ violation in any system through the exchange of 


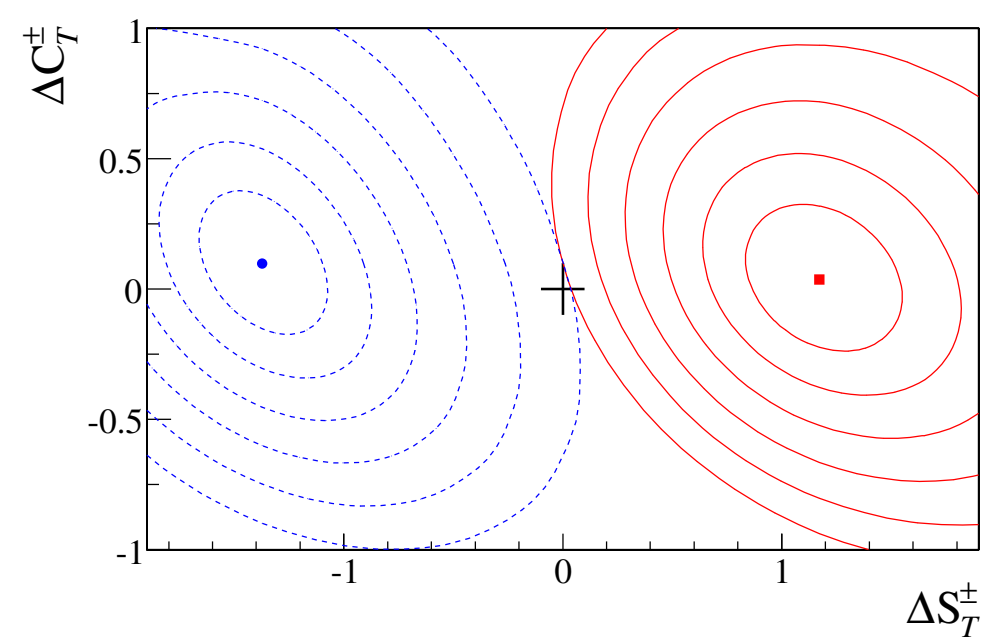

Figure 3. Central values and contours for 1-CL up to 6 standard deviations for the pairs of $T$-asymmetry parameters $\left(\Delta C_{T}^{+}, \Delta S_{T}^{+}\right)$(blued point and dashed lines) and $\left(\Delta C_{T}^{-}, \Delta S_{T}^{-}\right)$(red square and continuous lines). The "+" symbol at $\left(\Delta C_{T}^{ \pm}=\Delta S_{T}^{ \pm}=0\right)$ represents the $T$-invariance point.

initial and final states in transitions that can only be connected by a $T$-symmetry transformation. This result is independent from assumptions on the $C P T$ symmetry.

\section{Searches for a light CP-odd Higgs}

Practically all theoretical models for Physics beyond the SM include a number of additional Higgs bosons; the most popular, the MSSM, introduces 5 more Higgs, one of which, called the A, is CP odd. The next extension, the NMSSM introduces more Higgs, including a CP odd singlet. This singlet would mix with the MSSM A to give the $A^{0}$. It has been shown that this model is allowed by the LEP limits if the $A^{0}$ is light, with a mass less than twice the mass of the b quark. If this is the case, it could be produced in the radiative decays of the $\mathrm{Y}(\mathrm{nS})$ states and be seen by BABAR, provided that the branching ratios are large enough. The Higgs boson decay pattern depends on its mass and couplings, as well as the NMSSM particle spectrum. In the absence of a light neutralino, the $A^{0}$ decays predominantly into a pair of muons below $2 m_{\mu}$, while $\tau^{+} \tau^{-}$and hadronic final states become significant above this threshold. There is enough parameter space that BABAR could cover, to make a search worthwhile, and BABAR has performed searches for a light CP-odd Higgs boson in a variety of decay channels.

In the last months of running BABAR has collected large amounts of data at the $\Upsilon(2 S)$ and $\Upsilon(3 S)$ resonances: $99 \times 10^{6} \Upsilon(2 S)$ events and $122 \times 10^{6} \Upsilon(3 S)$. A clean sample of $\Upsilon(1 S)$ mesons is obtained by reconstructing the transitions $\Upsilon(3 S) \rightarrow \pi^{+} \pi^{-} \Upsilon(1 S)$. The signal topology consists of two oppositely-charged tracks originating from the interaction point and having missing mass equal to the $\Upsilon(1 S)$. With this method we have collected a very clean sample of about 20 million $\Upsilon(1 S)$. practically with no background from $e^{+} e^{-}$continuum. Using these data BABAR has performed searches for a light $C P$-odd Higgs boson in a variety of decay channels. These measurements are discussed in the next paragraphs, and the results are summarized in Table 3. 


\subsection{Search for $\Upsilon(1 S) \rightarrow \gamma A^{0}, A^{0} \rightarrow \mu^{+} \mu^{-}$}

To search for the $A^{0}$ decay into muon pairs we select events in the $\Upsilon(2 S)$ and $\Upsilon(3 S)$ data; we require only one photon and 4 charged tracks, two of which must be pions recoiling against a $\Upsilon(1 S)$, and at least one of the others must be identified as a muon. We require the kinematics to be compatible with this decay chain, and all tracks to originate from the interaction point. The signal would be a narrow peak in the dimuon invariant mass, whose resolution is better than $10 \mathrm{MeV}$.

The $\Upsilon(2,3 S)$ candidates are then fit, constraining their CM energies to the total beam energy, and imposing a common vertex for the tracks. A series of unbinned likelihood fits to the dimuon mass distribution is performed to extract the signal. No evidence of $A^{0}$ is observed; $90 \%$ confidence level (CL) limits on the branching fractions are established at the level of $(0.26-8.3) \times 10^{-6}$ for $0.212<m_{A^{0}}<9.3 \mathrm{GeV}$ [7]. The limits as a function of the $A^{0}$ mass are shown in the left side of Figure 3.1, together with limits on the product $B\left(A^{0} \rightarrow \mu^{+} \mu^{-}\right) f_{\gamma}^{2}$, where $f_{\gamma}^{2}$ denotes the effective coupling of the $b$ quark to the $\Upsilon$ meson $[8,9]$.
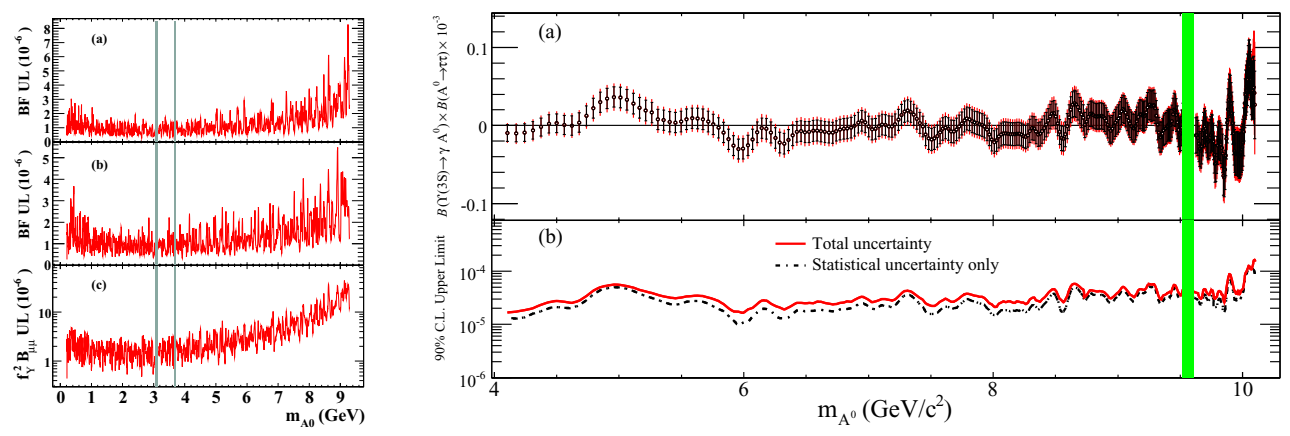

Figure 4. Left: $90 \% \mathrm{CL}$ upper limit on the $\Upsilon(3 S) \rightarrow \gamma A^{0}, A^{0} \rightarrow \mu^{+} \mu^{-}$(top) and $\Upsilon(2 S) \rightarrow \gamma A^{0}, A^{0} \rightarrow \mu^{+} \mu^{-}$ branching fractions (middle) as a function of the $A^{0}$ mass derived by BABAR. The limits on the product $B\left(A^{0} \rightarrow\right.$ $\left.\mu^{+} \mu^{-}\right) f_{\gamma}^{2}$, are also shown (bottom). The $J / \psi$ and $\psi(2 S)$ regions (solid bands) are excluded from the search. Right: Product of branching fraction $\Upsilon(3 S) \rightarrow \gamma A^{0}, A^{0} \rightarrow \tau^{+} \tau^{-}$(top) and the corresponding 90\% CL upper limit (bottom) as a function of the $A^{0}$ mass set by $B A B A R$. The region corresponding to $\chi_{b J}(2 P) \rightarrow \gamma \Upsilon(1 S)$ transitions (shaded band) is excluded.

\subsection{Search for $\Upsilon(1 S) \rightarrow \gamma A^{0}, A^{0} \rightarrow \tau^{+} \tau^{-}$}

In principle the analysis is the same as for muon pairs, but is more complicated because of the presence of neutrinos. We require each $\tau$ to decay in one charged track, and at least one of the twos into a lepton; we feed all the information in the event to a neural network to select the candidate events. We have to separate the sample on two parts, for lower and higher of $A^{0}$ mass because the background to the photon is quite different, and we look for peaks in the missing mass against the two pions and the photon. A set of eight kinematic and angular variables are used to further suppress the background, which arises mainly from radiative $\tau$ production and two-photon processes. The signal yield is extracted as a function of $m_{A^{0}}$ by a simultaneous fit to the photon energy distribution of the ee,$\mu \mu \gamma$ and $e \mu \gamma$ samples. We test about 200 mass hypotheses, the largest fluctuations we find has a significance 
of $3 \sigma$; from pseud0-experiments we find that the probability to have a fluctuation of more than $3 \sigma$ is $7.5 \%$, so again we conclude that the search is negative. Since no excess is seen, $90 \%$ CL limits on the branching fraction are set at the level of $(1.5-16) \times 10^{-5}$ in the interval $4.03<m_{A^{0}}<10.1 \mathrm{GeV}[10]$, as shown in the right side of Figure 3.1.

\subsection{Search for $\Upsilon(2 S, 3 S) \rightarrow \gamma A^{0}, A^{0} \rightarrow$ hadrons}

The hadronic final states are selected from fully reconstructed the $A^{0} \rightarrow$ hadrons decays. The highest energy photon of the event is identified as the radiative photon from the $\Upsilon(n S)$ decay; the $A^{0}$ candidate is then constructed by adding the four-momenta of the remaining particles, constraining the $A^{0}$ decays products to originate from the interaction point to improve the resolution. The signal yield is obtained by fitting the candidate mass spectrum in the range $0.3-7.0 \mathrm{GeV}$ in steps of $1 \mathrm{MeV}$. The results are compatible with the null hypothesis; limits on the branching fraction are therefore set in the range $(0.1-8) \times 10^{-5}$ with $90 \%$ confidence level [11].

\subsection{Search for $\Upsilon(1 S) \rightarrow \gamma A^{0}, A^{0} \rightarrow$ invisible}

Searches have also been performed by BABAR on $A^{0}$ decaying into invisible, i.e. with nothing seen in the detector. In the search for $\Upsilon(2 S) \rightarrow \pi^{+} \pi^{-} \Upsilon(1 S), \Upsilon(1 S) \rightarrow \gamma A^{0}, A^{0} \rightarrow$ invisible the final state is characterized by a pair of low-momentum tracks, a single energetic photon, and large missing energy and momentum. Additional criteria on the photon and the extra neutral energy in the event are applied to further suppress contributions from electron bremsstrahlung, radiative hadronic $\Upsilon(1 S)$ decays and two-photon processes in which particles escape undetected. The signal is extracted by a series of bidimensional unbinned likelihood fits to the dipion recoil mass and the missing mass squared for both two-body decays, $\Upsilon(1 S) \rightarrow \gamma A^{0}, A^{0} \rightarrow$ invisible, and non-resonant three-body processes, $\Upsilon(1 S) \rightarrow \gamma A^{0}, A^{0} \rightarrow \chi \bar{\chi}$. Values of $m_{A^{0}}$ and $m_{\chi_{0}}$ are probed over $0<m_{A}<9.2 \mathrm{GeV}$ and $0 \leq m_{\chi_{0}} \leq 4.5 \mathrm{GeV}$, respectively. No significant signal is found; $90 \% \mathrm{CL}$ limits $B(\Upsilon(1 S) \rightarrow$ $\gamma A^{0}, A^{0} \rightarrow$ invisible $)<(1.9-37) \times 10^{-6}$ and $B\left(\Upsilon(1 S) \rightarrow \gamma A^{0}, A^{0} \rightarrow \chi^{0} \bar{\chi}^{0}\right)<(1.9-37) \times 10^{-6}$ are set [12]. These limits are displayed in Figure 5 as a function of the $A^{0}$ and $\chi^{0}$ masses.
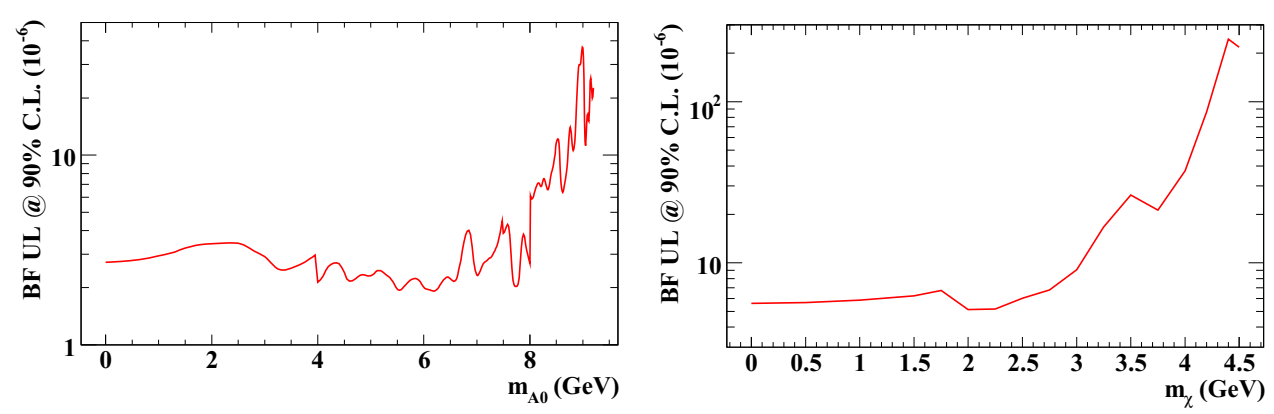

Figure 5. 90\% CL upper limits on the $\Upsilon(1 S) \rightarrow \gamma A^{0}, A^{0} \rightarrow$ invisible (left) and $\Upsilon(1 S) \rightarrow \gamma A^{0}, A^{0} \rightarrow \chi \bar{\chi}$ (right) branching fractions as a function of the $A^{0}$ and $\chi$ masses set by BABAR.

A search for $\Upsilon(3 S) \rightarrow \gamma A^{0}, A^{0} \rightarrow$ invisible was also performed selecting events containing a single energetic photon with no additional activity in the detector. The background arises mainly 


\begin{tabular}{lrr}
\hline Mode & Mass range $(\mathrm{GeV})$ & BF upper limit (90\% CL) \\
\hline$\Upsilon(2 S, 3 S) \rightarrow \gamma A^{0}, A^{0} \rightarrow \mu^{+} \mu^{-}$ & $0.21<m_{A}<9.3$ & $(0.3-8.3) \times 10^{-6}$ \\
$\Upsilon(3 S) \rightarrow \gamma A^{0}, A^{0} \rightarrow \tau^{+} \tau^{-}$ & $4.0<m_{A}<10.1$ & $(1.5-16) \times 10^{-5}$ \\
$\Upsilon(2 S, 3 S) \rightarrow \gamma A^{0}, A^{0} \rightarrow$ hadrons & $0.3<m_{A}<7.0$ & $(0.1-8) \times 10^{-5}$ \\
$\Upsilon(1 S) \rightarrow \gamma A^{0}, A^{0} \rightarrow \chi \bar{\chi}$ & $m_{\chi}<4.5 \mathrm{GeV}$ & $(0.5-24) \times 10^{-5}$ \\
$\Upsilon(1 S) \rightarrow \gamma A^{0}, A^{0} \rightarrow$ invisible & $m_{A}<9.2 \mathrm{GeV}$ & $(1.9-37) \times 10^{-6}$ \\
$\Upsilon(3 S) \rightarrow \gamma A^{0}, A^{0} \rightarrow$ invisible & $m_{A}<9.2 \mathrm{GeV}$ & $(0.7-31) \times 10^{-6}$ \\
$\Upsilon(1 S) \rightarrow \gamma A^{0}, A^{0} \rightarrow g \bar{g}$ & $m_{A}<9.0 \mathrm{GeV}$ & $10^{-6}-10^{-2}$ \\
$\Upsilon(1 S) \rightarrow \gamma A^{0}, A^{0} \rightarrow s \bar{s}$ & $m_{A}<9.0 \mathrm{GeV}$ & $10^{-5}-10^{-3}$ \\
\hline
\end{tabular}

Table 3. Results of light Higgs boson searches performed by the BABAR Collaboration.

from $e^{+} e^{-} \rightarrow \gamma \gamma$, radiative Bhabha, and two-photon fusion events. The $A^{0}$ yield is extracted by a series of unbinned likelihood fits to the photon energy distribution for $0<m_{A^{0}}<7.8 \mathrm{GeV}$. No excess is seen, and limits on the branching fraction at the level of $(0.7-31) \times 10^{-6}$ are derived with $90 \%$ confidence level [13].

\subsection{Search for $\Upsilon(1 S) \rightarrow \gamma A^{0}, A^{0} \rightarrow g \bar{g}$ or $s \bar{s}$}

A recent search was performed by BABAR for $\left.\Upsilon(2 S) \rightarrow \pi^{+} \pi^{-}-\Upsilon(1 S)\right), \Upsilon(1 S) \rightarrow \gamma A^{0}, A^{0} \rightarrow$ $g \bar{g}(\operatorname{or} s \bar{s})$. Selected events with final states consisting of three or more light adrons, in addition to the two pions from the $\Upsilon(2 S)$ decay, and the radiative photon. A total of 26 final states composed of light hadrons were studied, including some containing at least a kaon pair, which were assigned to the $A^{0} \rightarrow s \bar{s}$ decay. The main background is due to $\Upsilon(1 S)$ decay to $g g g$, where one of the $\pi^{0}$ of the hadronization decays to photons, one of which is mistaken for the radiative one. The $A^{0}$ mass range explored is 0.5 to $9 \mathrm{GeV}$. We observe no signals [14]in the hadronic invariant mass spectra, and set upper limits at $90 \%$ CL limits on the product branching for $\Upsilon(1 S) \rightarrow \gamma A^{0}, A^{0} \rightarrow g \bar{g}$ from $10^{-6}$ to $10^{-2}$ ; for the branghing ratio $\Upsilon(1 S) \rightarrow \gamma A^{0}, A^{0} \rightarrow s \bar{s}$ the corresponding limits are from $10^{-5}$ to $10^{-3} \mathrm{We}$ do not observe a NMSSM $A^{0}$ or any narrow hadronic resonance.

\section{Search for light dark matter}

We have now overwhelming astrophysical evidence for dark matter with several possibly related anomalies observed. There is more than one explanation, of course, and most models introduce a new dark force mediated by a new gauge boson with a mass around a GeV. Dark matter particles are expected at the $\mathrm{TeV}$ scale, but the lightest particles in which they would annihilate could be pairs of light dark bosons, which subsequently could only decay into lepton pairs, or scatter. This light hidden sector is poorly constrained, and it is worth exploring the possibility that these particles are produced at accelerators. B-factories offer a low background environment, so signatures of dark particles at the $\mathrm{MeV} / \mathrm{GeV}$ scale, should not escape detection, and a discovery would allow to probe their structure. The 2 sectors could interact via kinematical mixing, and the value of the mixing parameter would be the key to a possible detection. The dark photon, the equivalent of the e.m. photon, could have a mass of the order from $\mathrm{MeV}$ to $\mathrm{GeV}$, and would couple to the SM fermions with a charge $\epsilon$. The preferred value for $\epsilon$ is from $10^{-5}$ to $10^{-3}$ and several experiments have already put limits. The hidden boson masses are usually generated via the Higgs mechanism, adding hidden Higgs bosons $\left(h^{\prime}\right)$ to the theory. 
A minimal hidden sector model includes a single hidden photon and a Higgs boson [15]. Additional Higgs and gauge bosons appear in more complex setups [16]. The coupling strength between the SM and the hidden sector, $\epsilon$, can naturally be as large as $10^{-4}-10^{-2}$ [15], opening the possibility of probing a light hidden sector at low-energy $e^{+} e^{-}$colliders. A search for dark photon and associated scalar boson has been performed at BABAR in the range $0.8<m_{h^{\prime}}<10.0 \mathrm{GeV}$ and $0.25<m_{A^{\prime}}<3.0 \mathrm{GeV}$, with the constraint $m_{h^{\prime}}>2 m_{A^{\prime}}$ [17]

\subsection{Search for a hidden photon}

Hidden photons can be readily produced in $e^{+} e^{-} \rightarrow \gamma A^{\prime}, A^{\prime} \rightarrow l^{+} l^{-}(l=e, \mu)$ interactions, and be detected as resonances in the $l^{+} l^{-}$spectrum. This signature is similar to that of light $C P$-odd Higgs production in $e^{+} e^{-} \rightarrow \Upsilon(2 S, 3 S) \rightarrow \gamma \mu^{+} \mu^{-}$[7], and searches for this final state have been reinterpreted as searches for hidden photon production [18]. These limits are shown in Fig. 6, together with the constraints derived from the measurement of the muon anomalous magnetic moment, and searches at lowenergy $e^{+} e^{-}$colliders and fixed-target experiments [18],Giovannella:2011nh,Abrahamyan:2011gv. Values of the mixing strength down to $10^{-3}-10^{-2}$ are excluded for $0.212<m_{A^{\prime}}<9.3 \mathrm{GeV}$. Future analyses of the $e^{+} e^{-} \rightarrow \gamma l^{+} l^{-}(l=e, \mu)$ final states are expected to probe values of the mixing strength down to $10^{-3}$ in the range $0.01<m_{A^{\prime}}<10 \mathrm{GeV}$, significantly improving existing bounds.

Other measurements may be reinterpreted as constraints on hadronic hidden photon decays. These include searches for peaks in inclusive $e^{+} e^{-} \rightarrow \gamma+$ hadrons production [11], as well as exclusive hadronic final states, such as $e^{+} e^{-} \rightarrow \gamma \pi^{+} \pi^{-}[13]$.

\subsection{Search for a light dark Higgs}

Extensions of hidden sectors to a non-Abelian groups introduce additional hidden gauge bosons to the theory. The detailed phenomenology is model dependent, but heavy hidden bosons decay to lighter hidden states if kinematically accessible, while the lightest bosons are metastable and decay to SM fermions via their mixing with the hidden photon [16].

The Higgsstrahlung process, $e^{+} e^{-} \rightarrow A^{\prime} h^{\prime}, h^{\prime} \rightarrow A^{\prime} A^{\prime}$, offers another promising gateway to hidden sectors, since it is one of the few processes suppressed by a single power of the mixing strength, and the background is expected to be small. The event topology depends on the boson mass: Higgs bosons heavier than two hidden photons decay promptly, while their lifetime becomes large enough to escape undetected in the regime $m_{h^{\prime}}<m_{A^{\prime}}$.

$B A B A R$ performed a search for a hidden Higgs boson in Higgsstrahlung production in the prompt decay regime based on $521 \mathrm{fb}^{-1}$ of data [17]. The measurement is performed in the range $0.8<$ $m_{h^{\prime}}<10.0 \mathrm{GeV}$ and $0.25<m_{A^{\prime}}<3.0 \mathrm{GeV}$, with the constraint $m_{h^{\prime}}>2 m_{A^{\prime}}$. The signal is either fully reconstructed into lepton or pion pairs (exclusive mode), or partially reconstructed (inclusive mode). Only two of the three hidden photons are reconstructed in the latter case, and the four-momentum of the remaining hidden photon is identified to that of the recoiling system. The topology of the exclusive modes consists of six tracks having an invariant mass close to the center-of-mass energy, forming three hidden photon candidates with equal masses. Inclusive modes are first identified by selecting two dileptonic resonances with similar mass, and requiring the mass of the recoiling system to be compatible with the Higgsstrahlung hypothesis.

No significant signal is observed. Using uniform priors in the cross-section, upper limits on the $e^{+} e^{-} \rightarrow A^{\prime} h^{\prime}, h^{\prime} \rightarrow A^{\prime} A^{\prime}$ cross section are derived as a function of the hidden Higgs and hidden photon masses, including systematic uncertainties.

These results are translated into limits on the product $\alpha_{D} \epsilon^{2}$, and displayed in Fig. 7 as a function of the hidden photon mass for selected values of the hidden Higgs boson mass. Values down to 


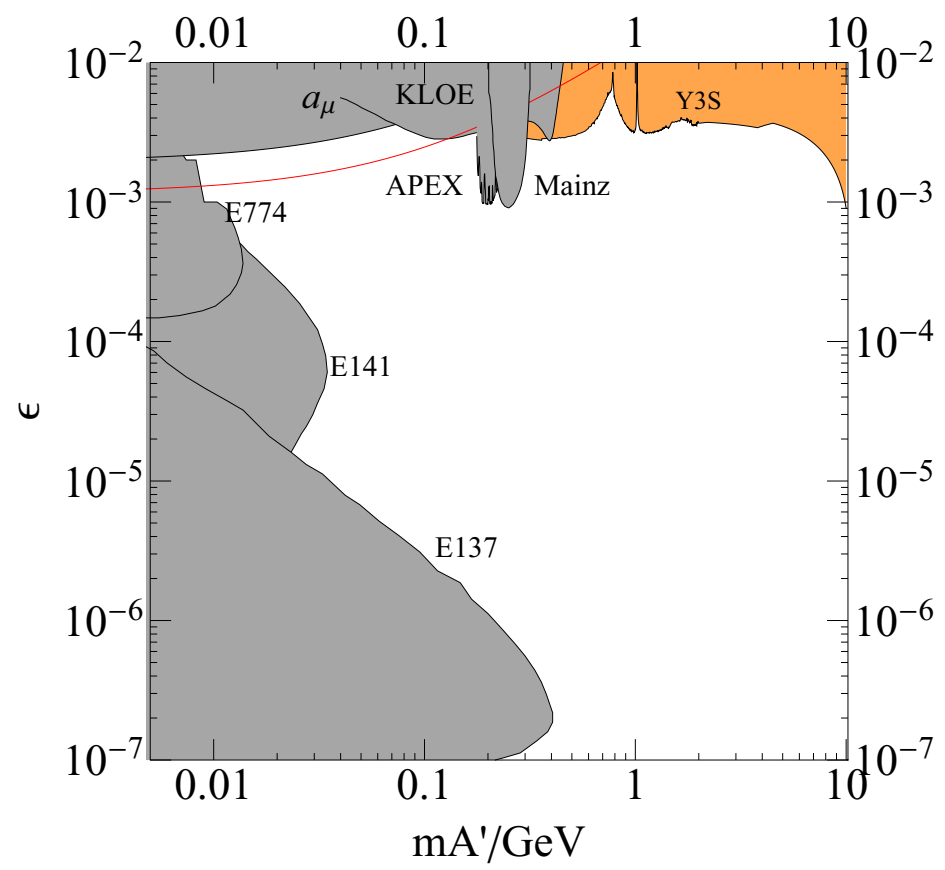

Figure 6. Constraints on the mixing parameters, $\epsilon$, as a function of the hidden photon mass derived from searches in $\Upsilon(2 S, 3 S)$ decays at $B A B A R$ (orange shading) and from other experiments [18-20] (gray shading). The red line shows the value of the coupling required to explain the discrepancy between the calculated and measured anomalous magnetic moment of the muon [?].

$10^{-10}-10^{-8}$ are excluded for a large range of hidden photon and hidden Higgs masses, assuming prompt decays. Assuming $\alpha_{D}=\alpha \simeq 1 / 137$, limits on the mixing strength in the range $10^{-4}-10^{-3}$ are derived, an order of magnitude smaller than the current experimental bounds extracted from direct photon production in this mass range.

\section{Summary}

More than 5 years after completion of the data taking, the BABAR collaboration is still very active. The great amount of data collected is stimulating new ideas. The T violation measurement is a first and constitues a beautiful proof of the CPT theorem. Searches for exotic particles have not given positive results, but have contributed to considerably narrow the parameters space. One of the hot topics in Particles Physics is now dark matter: recent evidence has suggested that dark matter might contain a $\mathrm{MeV}-\mathrm{GeV}$ scale component. Thanks to their large luminosities, $B$ factories provide an ideal environment to probe for such a possibility, complementing direct detection and satellite experiments. No sign of light dark matter has been observed so far, but several new analyses are going on and we still hope for surprises. A big step forward is expected with the atart of the Super flavor factory at KEK: BELLE-II is expected to increase the sensitivity of these searches by a factor $10-100$. 


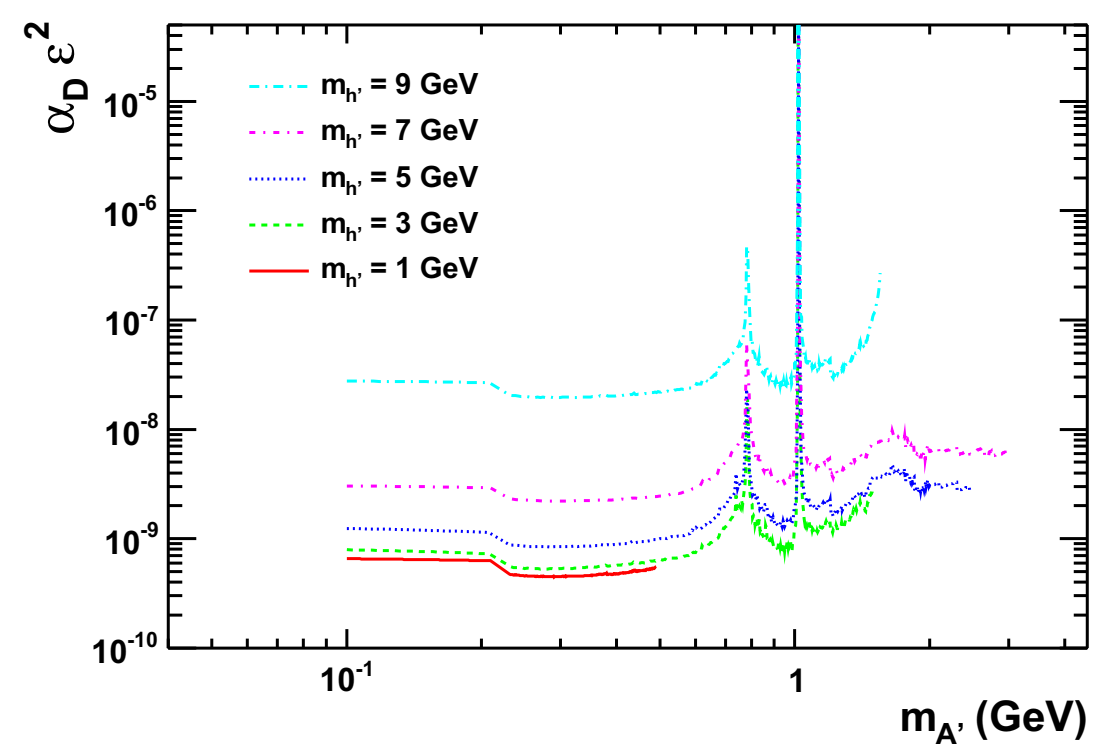

Figure 7. The $90 \%$ confidence level upper limit on the product $\alpha_{D} \epsilon^{2}$ as a function of the hidden photon mass $\left(m_{A^{\prime}}\right)$ for selected values of hidden Higgs boson masses $\left(m_{h^{\prime}}\right)$.

\section{Acknowledgments}

The results that I have presented here have been obtained by the BABAR Collaboration; I have to thank all my BABAR colleagues and I am grateful in particular to Giuseppe Finocchiaro and Bertrand Echenard for their help in preparing this manuscript. I would like to thank the organizers for inviting me, and for the excellent work in running an interesting and stimulating Conference and a very pleasurable stay in the wonderful setting of Kolymbari

\section{References}

[1] B. Aubert et al. (BABARCollaboration), The BABARdetector. Nuclear Instruments and Methods in Physics Research A 479 (2002) 1-116.

[2] B. Aubert et al. (BABARCollaboration), Measurement of time-dependent CP asymmetry in $B \rightarrow$ $c \bar{c} K^{(*) 0}$ decays. Phys. Rev. D 79, 072009 (2009).

[3] CPLEAR Collaboration: A. Angelopoulos et al., First direct observation of time-reversal noninvariance in the neutral-kaon system. Phys. Lett. B444 (1998) 52.

[4] M. Bañuls and J. Bernabeu, CP, T and CPT versus temporal asymmetries for entangled states of the $B_{d}$ system. Phys. Lett. B 464 (1999) 117.

[5] J. Bernabeu, F. Martinez-Vidal, P. Villanueva-Perez, Time reversal violation from the entangled $B^{0} \bar{B}^{0}$ system. JHEP 1208, 064 (2012).

[6] J.P. Lees et al. (BABARCollaboration), Observation of Time-Reversal violation in the $B^{0}$ meson system. Phys. Rev.Lett. 109, 211801 (2012).

[7] B. Aubert et al. [BABAR Collaboration], Phys. Rev. Lett. 103, 081803 (2009).

[8] P. Nason, Phys. Lett. B 175, 223 (1986). 
[9] F. Wilczek, Phys. Rev. Lett. 39, 1304 (1977).

[10] B. Aubert et al. [BABAR Collaboration], Phys. Rev. Lett. 103, 181801 (2009).

[11] J. P. Lees et al. [BABAR Collaboration], Phys. Rev. Lett. 107, 221803 (2011).

[12] P. del Amo Sanchez et al. [BABAR Collaboration], Phys. Rev. Lett. 107, 021804 (2011).

[13] B. Aubert et al. [BABAR Collaboration], Phys. Rev. Lett. 103, 231801 (2009).

[14] J.P. Lees et al.Phys. Rev. D 88, 031701 (2013)

[15] B. Batell, M. Pospelov and A. Ritz, Phys. Rev. D 79, 115008 (2009).

[16] R. Essig, P. Schuster and N. Toro, Phys. Rev. D 80, 015003 (2009).

[17] J. P. Lees [BABAR Collaboration], arXiv:1202.1313 [hep-ex].

[18] J. D. Bjorken, R. Essig, P. Schuster and N. Toro, Phys. Rev. D 80,075018 (2009) and references therein.

[19] S. Giovannella, J. Phys. Conf. Ser. 335, 012067 (2011).

[20] S. Abrahamyan et al. [APEX Collaboration], Phys. Rev. Lett. 107, 191804 (2011). 